\title{
An Implicit Inversion Method for Estimating Boundary Conditions in Hydrologic Optics
}

\author{
M.R. RETAMOSO ${ }^{1}$, Departamento de Matemática, FURG; PROMEC - UFRGS \\ M.T. de VILHENA², PROMEC - UFRGS, Rio Grande do Sul, Brazil \\ H.F. de CAMPOS VELHO 3 , F.M. RAMOS, Laboratório Associado de Com- \\ putação e Matemática Aplicada (LAC), Instituto Nacional de Pesquisas Espaciais \\ (INPE), Cx.P. 515, 12201-970 São José dos Campos, SP, Brazil.
}

\begin{abstract}
An important topic in radiative process is the estimation of some characteristcs of the media and/or other conditions of the problem. This estimation process can be formulated as an inverse problem. This work presents an estimation of boundary conditions for natural waters, where the forward problem is solved by LTSn method. The reconstruction is made using radiance. The inverse methodology was tested with data corrupted with Gaussian noise, where the Thikhonov regularization was used.
\end{abstract}

\section{Introduction}

The direct or forward radiative transfer problem in hydrologic optics, in the steady state, involves the determination of the radiance distribution in a body of water given known boundary conditions, inherent optical properties (IOP): the absortion, scattering coefficients and phase function, and source term. The corresponding inverse radiative transfer problem arises when physical properties, internal light sources and/or boundary conditions must be estimated from radiometric measurements of the underwater light fields. In the last decades, the development of inversion methodologies for radiative transfer problems has been an important research topic in many branches of science and engineering (McCormick, [3]). Particularly in oceanography, the estimation of bioluminescence sources from light-emitting marine organisms - an issue of great relevance in the study of the biological-optical processes in the oceans - has been the subject of some recent works (Yi, Sanchez and McCormick, [13]), well as the unified estimation of the IOP's and the source term (Tao, McCormick and Sanchez, [11]).

\footnotetext{
${ }^{1}$ mario@lula.dmat.furg.br

2 vilhena@cesup.ufrgs.br

${ }^{3}$ haroldo@lac.inpe.br

${ }^{4}$ fernando@lac.inpe.br
} 
Here the inverse model is an implicit technique for function estimation from in situ radiometric measurements. The algorithm is formulated as a constrained nonlinear optimization problem, in which the direct problem is iteratively solved for successive aproximations of the unknown parameters. Iteration proceeds until an objective-function, representing the least-squares fit of model results and experimental data added to a regularization term, converges to a specified small value (Chalhoub and Campos Velho, [2]; Stephany et all., [7, 8, 9]). The associated direct problem is tackled with the LTSn method (Segatto and Vilhena, [10]). This model solves numerically the time-independent, one-dimensional radiative transfer equation in natural water bodies using an analytical solution of the discrete ordinate equations ( $S_{N}$ equations).

\section{Direct Model: LTSn for Solving Radiative Trans- fer Equation}

The radiative transfer equation, for a given wavelength, can be expressed as

$$
\mu \frac{d L(\zeta, \xi)}{d \zeta}=-L(\zeta, \xi)+\omega_{0}(\zeta) \int_{\Xi} L\left(\zeta, \xi^{\prime}\right) \beta\left(\xi^{\prime} \rightarrow \xi\right) d \xi^{\prime}+S(\zeta, \xi)
$$

where $L$ is the radiance, $\beta$ is the scattering phase function, $\omega_{0}=b / c$ is the single scattering albedo, $c=a+b$ is the beam attenuation coefficient, $a$ and $b$ are respectively the absortion and scattering coefficients, $\xi^{\prime}\left(\theta^{\prime}, \phi^{\prime}\right)$ and $\xi(\theta, \phi)$ are the incident and scattered directions for an infinitesimal beam, $\theta$ is the polar angle, $\phi$ is the azimuthal angle, $S$ is the source term, and $\mu=\cos (\theta)$.

The general problem (2.1) can be transformed to a system of equations with azimuthal symmetry, assumning a Fourier decomposition for azimuthal variable as showed by Chandrasekhar in 1950. From this consideration, the radiative equation, with depencency of the polar angle only, can be rewritten as (the underscript denoting the azimuthal dependence is omitted)

$$
\mu \frac{d L(\zeta, \mu)}{d \zeta}+L(\zeta, \mu)=\omega_{0} \int_{-1}^{1} \Theta\left(\mu^{\prime} \rightarrow \mu\right) L\left(\zeta, \mu^{\prime}\right) d \mu^{\prime}+S(\zeta, \mu)
$$

with following boundary conditions

$$
\begin{cases}L(0, \mu)=f_{1}(\mu) & \text { at } \mu>0 \\ L\left(\zeta_{0}, \mu\right)=f_{2}(\mu) & \text { at } \mu<0\end{cases}
$$

The discrete ordinate technique is a colocation method, where the integral term in Eq. (2.2) is approximated by a Gauss-Legendre quadrature for a $N_{\mu}$ finite number of polar angles. For simplicity, all IOP's are considered space-independent. Therefore, the integro-differential equation (2.2) becomes in a system of differential equations. Expressing this system in matrix form

$$
\frac{d \mathbf{L}(\zeta)}{d \zeta}=A \mathbf{L}(\zeta)+\mathbf{S}(\zeta)
$$


with discrete boundary conditions

$$
\begin{aligned}
\mathbf{L}^{+}(0) & =\left[\begin{array}{llll}
f_{1}\left(\mu_{1}\right) & f_{1}\left(\mu_{2}\right) & \ldots & f_{1}\left(\mu_{N_{\mu}}\right.
\end{array}\right]^{T} ; \\
\mathbf{L}^{-}\left(\zeta_{0}\right) & =\left[\begin{array}{llll}
f_{2}\left(\mu_{1}\right) & f_{2}\left(\mu_{2}\right) & \ldots & f_{2}\left(\mu_{N_{\mu}}\right.
\end{array}\right]^{T} .
\end{aligned}
$$

The matrix entries of system (2.4) are as following

$$
A_{i j}= \begin{cases}\Theta_{i j}\left(u_{i} / \mu_{i}\right)-\mu_{i}^{-1} & \text { if } i=j \\ \Theta_{i j}\left(u_{j} / \mu_{i}\right) & \text { if } i \neq j\end{cases}
$$

being $u_{i}$ 's the weights of quadrature, $\mu_{i}$ the quadrature node, that is, the $i$-th root of a Legendre polynomial of order $N_{\mu}$, with $\mathbf{L}(\zeta)=\left[L\left(\zeta, \mu_{1}\right) L\left(\zeta, \mu_{2}\right) \ldots L\left(\zeta, \mu_{N_{\mu}}\right)\right]^{T}$ and $\mathbf{S}(\zeta)=\left[\begin{array}{llll}S\left(\zeta, \mu_{1}\right) / \mu_{1} & S\left(\zeta, \mu_{2}\right) / \mu_{2} & \ldots & S\left(\zeta, \mu_{N_{\mu}}\right) / \mu_{N_{\mu}}\end{array}\right]^{T}$. The discrete phase function is obtained from the expanding it by Legendre polynomial

$$
\Theta_{i j} \simeq \sum_{l=0}^{N_{\beta}} \beta_{l} P_{l}\left(\mu_{i}\right) P_{l}\left(\mu_{j}\right) .
$$

Matrix problem (2.4) is solved by applying the Laplace transform on space variable resulting in the following operational equation

$$
(s I-A) \widehat{\mathbf{L}}(s)=\mathbf{L}(0)+\widehat{\mathbf{S}}(s)
$$

where $\widehat{\mathbf{L}}(s)=\mathcal{L}\{\mathbf{L}(\zeta)\}$. The resolvent $B(\zeta)=\mathcal{L}^{-1}\left\{(s I-A)^{-1}\right\}=\sum_{n=1}^{N_{\mu}} P^{n} e^{r_{n} \zeta}$ is obtained analytically using the Heaviside expansion technique, yielding

$$
\mathbf{L}(\zeta)=B(\zeta) \mathbf{L}(0)+\int_{0}^{\zeta} B(\zeta-\tau) \mathbf{S}(\tau) d \tau
$$

Finally, the vector $\mathbf{L}(0)$ is calculated by solving the algebraic linear system:

$$
\left[\begin{array}{l}
\mathbf{L}^{+}\left(\zeta_{0}\right) \\
\mathbf{L}^{-}\left(\zeta_{0}\right)
\end{array}\right]=\left[\begin{array}{ll}
B_{11}\left(\zeta_{0}\right) & B_{12}\left(\zeta_{0}\right) \\
B_{21}\left(\zeta_{0}\right) & B_{22}\left(\zeta_{0}\right)
\end{array}\right]\left[\begin{array}{l}
\mathbf{L}^{+}(0) \\
\mathbf{L}^{-}(0)
\end{array}\right]+\left[\begin{array}{l}
\mathbf{H}^{+}\left(\zeta_{0}\right) \\
\mathbf{H}^{-}\left(\zeta_{0}\right)
\end{array}\right]
$$

denoting $H(\zeta) \equiv \int_{0}^{\zeta} B(\zeta-\tau) \mathbf{S}(\tau) d \tau$.

The exponential behavior of the solution, additioned to the fact that the eigenvalues $r_{n}$ increase in magnitude with $N_{\mu}$, implys in an adjust on the form (2.8). The difficulty mentioned can be avoided using a variable changing on $\zeta$ (Segatto and Vilhena, [10]):

$$
B(\zeta)=\sum_{n=1}^{N_{\mu} / 2} P^{n} e^{r_{n} \zeta}+\sum_{n=N_{\mu} / 2+1}^{N_{\mu}} P^{n} e^{r_{n} \zeta}=B^{+}(\zeta)+B^{-}(\zeta) ;
$$

which permits to write the solution as

$$
\mathbf{L}(\zeta)=B^{+}\left(\zeta-\zeta_{0}\right) \overline{\mathbf{L}}\left(\zeta_{0}\right)+B^{-}(\zeta) \overline{\mathbf{L}}(0)+\overline{\mathbf{H}}(\zeta)
$$

The convergence of the LTSn method was established using the $C_{0}$-semi group theory (Pazos and Vilhena, [5]). 


\section{Formulation for the Inverse Problem}

An overview of the recent inverse radiative transfer problems is found in McCormick [3]. In the present paper, we describe an implicit inversion technique for reconstruction of boundary conditions from in situ radiometric measurements.

The least squares approximation, in the sense of the minimum norm, can guarantee the existence of a solution, but it can be unstable in the presence of noise, a permanent feature in the experimental data. In order to have a robust inverse model, assuring that parameter variation are bounded to become the final solution physically acceptable, some a priori information must be added to the quadratic difference term. In general, this additional information associated to the inverse solution means smoothness.

Denoting by $\boldsymbol{p}=\left[p_{1}, p_{2}, \ldots, p_{N_{p}}\right]^{T}$ the unknown vector to be determined by the inverse analysis, the inverse radiative transfer problem can be formulated as a nonlinear constrained minimization problem,

$$
\min J(\boldsymbol{p}), l_{q} \leq p_{q} \leq u_{q}, q=1, \ldots, N_{p},
$$

where the lower and upper bounds $l_{q}$ and $u_{q}$ are chosen in order to allow the inversion to lie within some known physical limits, and the objective function is given by

$$
J(\boldsymbol{p})=\sum_{i=1}^{N_{m}}\left[L_{i}^{\operatorname{Exp}}-L_{i}^{\mathrm{Mod}}(\boldsymbol{p})\right]^{2}+\gamma \Omega[\boldsymbol{p}],
$$

being $N_{m}$ the number of measurement points in the water layer, $\Omega[\boldsymbol{p}]$ and $\gamma$ the operator and the parameter of regularization, and $L$ the radiometric quantity.

The present inverse problem adimits two procedures to find the boundary conditions (2.3) or (2.5). The unknown function can be solved as a parameter estimation approach, or a function estimation approach, where the functional form is not available. For practical purpose, a sampled function is considered:

$$
\boldsymbol{p}=\left[\begin{array}{l}
\boldsymbol{f}_{1} \\
\boldsymbol{f}_{2}
\end{array}\right]=\left[\begin{array}{llll}
f_{k}\left(\mu_{1}\right) & f_{k}\left(\mu_{2}\right) \ldots f_{k}\left(\mu_{N_{f}}\right)
\end{array}\right]^{T} \quad(k=1,2) .
$$

In the absence of an explicit solution, the optimization problem defined by Eq. (3.1) is iteratively solved by the quasi-newtonian optimization algorithm E04UCF from the NAG Fortran Library. This approach has been previously adopted with success in many applications. This routine minimizes an arbitrary smooth function subjected to constraints (simple bounds, linear or nonlinear constraints), using a sequential programming method.

\subsection{Tikhonov Regularization}

A well-known regularization technique proposed by Tikhonov (Tikhonov and Arsenin, [12]) can be expressed by

$$
\Omega[\boldsymbol{p}]=\sum_{k=0}^{N} \alpha_{k}\left\|\boldsymbol{p}^{(k)}\right\|_{2}^{2}
$$


where $\boldsymbol{p}^{(k)}$ denotes the $k$-th derivative (difference), and the parameters $\alpha_{k} \geq 0$. In this work, if $\alpha_{k}=\delta_{k j}$ (Kronecker's delta), i.e.,

$$
\Omega[\boldsymbol{p}]=\left\|f^{(j)}\right\|_{2}^{2}
$$

then the method is called the Tikhonov regularization of order- $j$ (Tikhonov-j). Particularly, the Tikhonov regularization of order zero will be referenced only as Tikhonov regularization.

\section{Numerical Results}

The performance of the inversion method presented in the previous section has been evaluated using synthetic radiometric data, i.e., data are generated by the same direct model used in the inverse solver for a single wavelength. The computational domain has been discretized into a vertical radiometric grid of $N_{z}=11$ nodes, ranging from 0 to $z_{\max }=4 \mathrm{~m}$. In all simulations, $\beta$ was given by an expantion in Legrendre polynomials of the one-term Henyey-Greenstein scattering phase function (Retamoso, [6]) expressed as follows:

$$
\beta(\cos \psi)=\frac{1}{4 \pi}\left(1-g^{2}\right)\left(1+g^{2}-2 g \cos (\psi)\right)^{-3 / 2} \simeq \sum_{l=0}^{N_{\mu}} \frac{(2 l+1)}{4 \pi} g^{l} P_{l}(\cos \psi)
$$

where $\psi$ is the scattering angle (formed by $\xi^{\prime}$ and $\xi$ directions) and $g=0.90$. The inherent optical properties were assumed to be constant, and Monterey bay water conditions, under sunlight and without wind, have been considered, taken from a similar work (Tao, McCormick and Sanchez 1994). At the sea surface, a cardioidal radiance distribution is taken for simulating the diffuse sunlight $\left(1 \mathrm{~W} / \mathrm{m}^{2} \mathrm{~nm}\right)$, and the source term was considered null.

The radiances are computed in $N_{\mu}=16$ points of quadrature, and $i=1,2, \ldots, N_{z}$ depths, defining a vertical grid of resolution $\Delta z=z_{\max } / N_{z}$. The measurements were done for uniformly spaced points, identified as grid-2. The synthetic experimental data were generate by the direct model added a Gaussian white noise with $10 \%$ of noise.

In order to identify the bottom boundary condition, two simulations were carried out. The first one, the inverse problem was solved without any regularization technique $(\alpha=0)$. Figure 1 displays the reconstruction without regularization, it is seen that the least square estimator procudes a wrong solution for the inverse problem. In a second simulation, the Tikhonov regularization of first-order was applied $\left(\gamma=2 \times 16^{-6}\right)$, and the estimation of boundary condition is shown in figure 2, where a good agreement between estimated and true solutions can be observed.

\section{Final Comments}

In the present paper, we have introduced a reconstruction technique of boundary conditions in natural waters from in situ radiance data. Assuming that the 


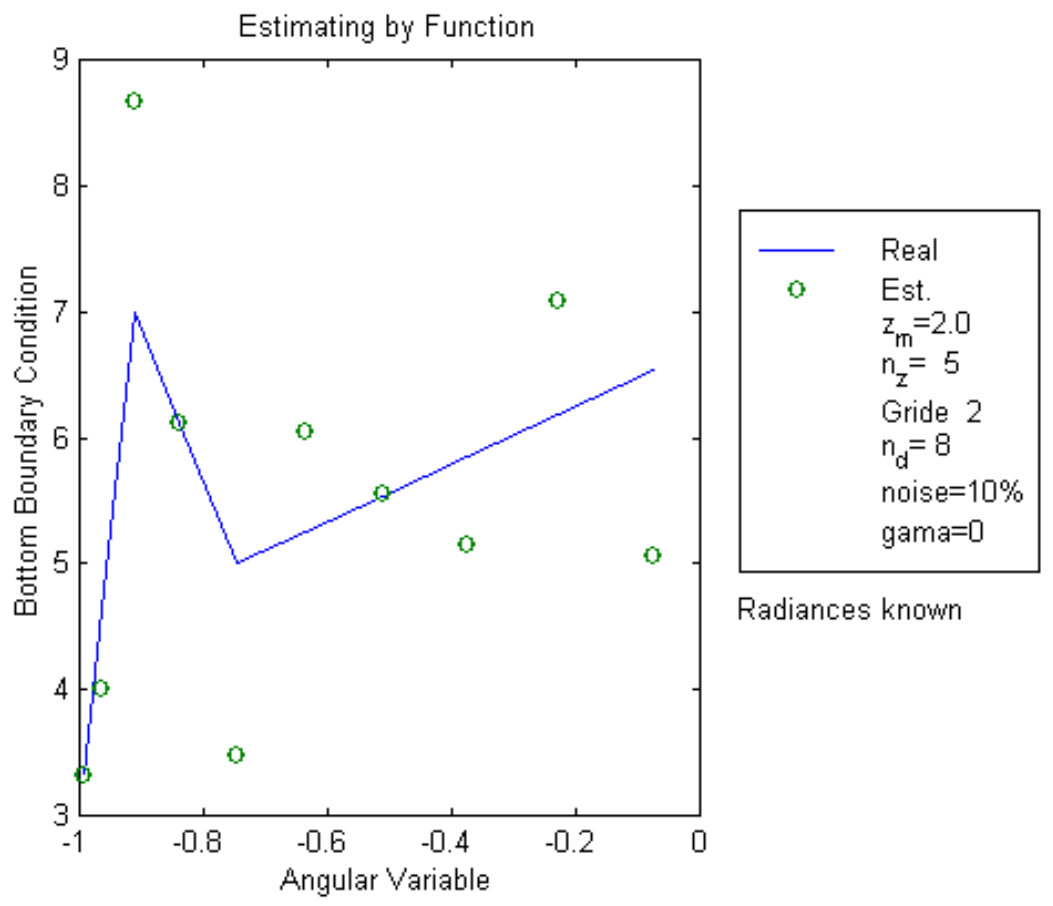

Figure 1: Reconstruction of bottom boundary conditions witout regularization.

unknown function can be sampled, the inverse problem was formulated as a nonlinear constrained optimization problem, and iteratively solved by a quasi-Newtonian minimization routine.

The proposed inversion technique has been tested yielding good numerical results. This metodology can also be applied for finding both boundary condition. The reconstruction of botton boundary condition was done because this case is more frequent in real world.

In a future work the inversion method described here will be used in a problem with different type of experimental data: different grid for measurements, and irradiances will be considered. It is also our intention to use a reconstruction by parameter approach, where the boundary condition is given by an expansion of certain base functions, in order to reduce the dimension of search space.

Acknowledgments: The authors thanks to the FAPESP, São Paulo State Foundation for partial finantial supporting this work through a Thematic Project grant (process 96/07200-8). 


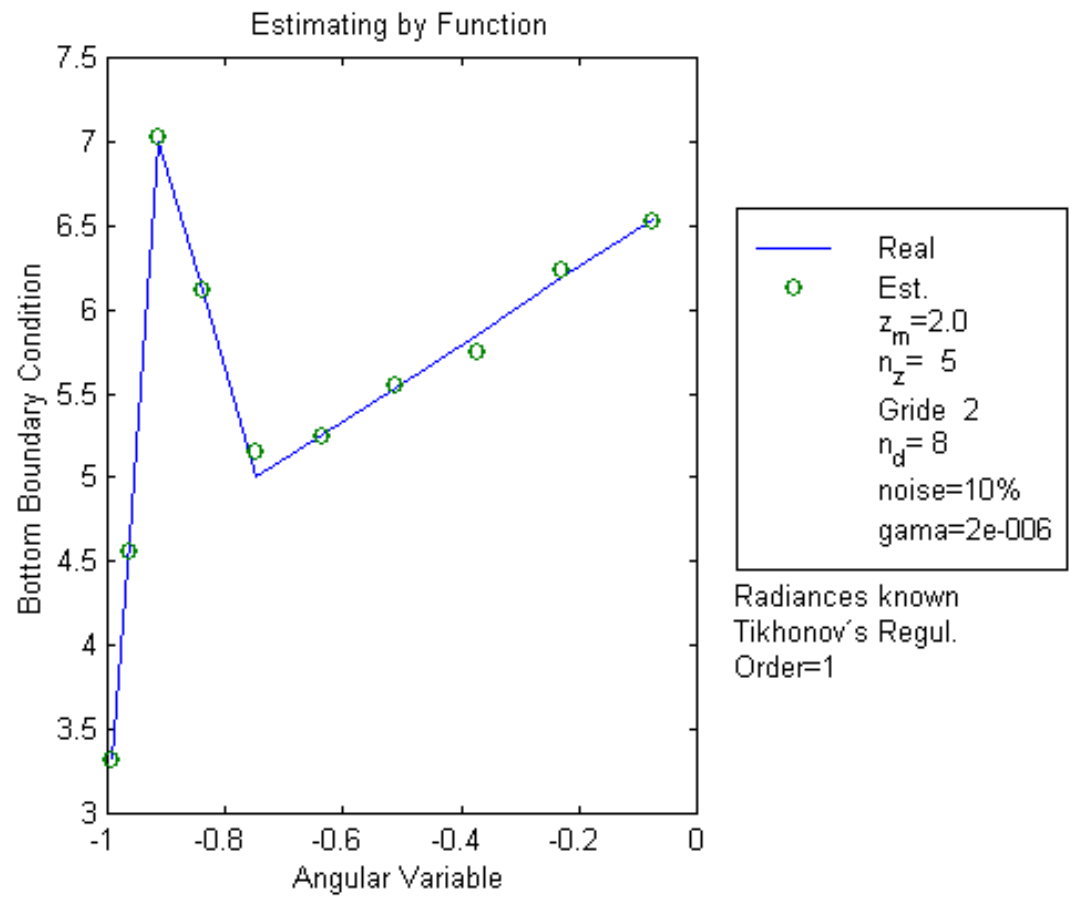

Figure 2: Reconstruction of bottom boundary condition with Tikhonov regularization.

\section{References}

[1] L.B. Barichello, M.T. Vilhena, Kerntecnik, 58 (1993), 182-184.

[2] E.S. Chalhoub, H.F. Campos Velho, Journal of Quantitative Spectroscopy \& Radiative Transfer (JQSRT), 1999 - submitted.

[3] N.J. McCormick, Nuclear Sci. and Eng., 112 (1992), 185-198.

[4] E04UCF NAG Fortran Library Mark 13, Oxford, UK, 1993.

[5] R.P. Pazos, M.T. Vilhena, Progress in Nuclear Energy, 34 (1999), 77-86.

[6] M.R. Retamoso, Obtenção de Fonte e de Condições de Contorno pelo Método LTSn em Transporte de radiação, Monography for Qualifing Examination, Graduation Course on Mechanical Engineering, PROMEC-UFRGS, Brazil, 1997.

[7] S. Stephany, F.M. Ramos, H.F. Campos Velho, C.D. Mobley, Computer Modeling and Simulation in Engineering (CMSE), 3 No. 3 (1998), 161-165. 
[8] S. Stephany, F.M. Ramos, H.F. Campos Velho, C.D. Mobley, JQSRT, 1999a accepted.

[9] S. Stephany, F.M. Ramos, H.F. Campos Velho, C.D. Mobley, Applied Optics, 1999b - submitted.

[10] C.F. Segatto, M.T. Vilhena, Annals of Nuclear Energy, 21 (1994), 701-710.

[11] Z. Tao, N.J. McCormick, R. Sanchez, Applied Optics, 33 (1994), 3265-3275.

[12] A.N. Tikhonov, V.Y. Arsenin, "Solutions of Ill-Posed Problems", Winston and Sons, 1977.

[13] H.C. Yi, R. Sanchez, N.J. McCormick, N.J., Applied Optics, 31 (1992), 822830. 